\title{
Raus aus der GOZ-Falle
}

Wie Sie den nebenstehenden Ausführungen des Kollegen Dr. Christian Öttl entnehmen können, besteht durch Fehlbewertungen in der Gebührenordnung für Zahnärzte (GOZ) 2012 häufig eine krasse Unterhonorierung von Leistungen. Bei mittlerweile (Stand 2016) mehr als 25 GebührenPositionen der GOZ 2012 werden bei gleichem Leistungsinhalt Honorare in Höhe der Sozialversicherung erst mit Steigerungsfaktoren von meist deutlich über 3,5 erreicht.

Die abweichende Vereinbarung ermöglicht eine Honorierung jenseits des ansonsten vorgeschriebenen Gebührenrahmens bis maximal Faktor 3,5. Gründe für abweichende Vereinbarungen liegen in einer Ausführungsqualität, die jenseits eines mittleren Leistungsstandards (BVerfG 1 BvR Az.1437/02, 25.10.2004) liegt. Nach wie vor gelten diese Aussagen der Verfassungsrichter, und sie gelten unter den aktuellen Verschiebungen der Honorarhöhe zwischen GKV und PKV verschärft:

„(...) Geht man davon aus, dass der 2,3-fache Steigerungssatz der Gebührenordnung der Vergütung entsprechender Leistungen zu Lasten der gesetzlichen Krankenversicherung entspricht [Anmerkung des Autors: eine Annahme von 2004, die heute bei über 70 Leistungen der GOZ nicht mehr gegeben ist!], besteht innerhalb des - ursprünglich deutlich weiter bemessenen - gesetzlichen Rahmens wenig Spielraum für die Berücksichtigung qualitativer Besonderheiten. Wo aber wegen des besonderen Aufwandes einer Leistung eine angemessene Vergütung durch den vorgegebenen Gebührenrahmen nicht mehr gewährleistet ist, bedarf es einer Öffnungsklausel, die im Einzelfall ein Abweichen von der Gebührenordnung erlaubt. Damit wird sichergestellt, dass dem Leistungserbringer nicht unangemessen niedrige Vergütungssätze oder von ihm abgelehnte Leistungsstandards zugemutet werden (...).“

\section{Honorarvereinbarung schriftlich treffen}

Die Honorarvereinbarung muss unbedingt nach persönlicher Absprache in jedem Einzelfall zwischen Zahnarzt und Patient (Zahlungspflichtigem) vor Erbringung der Leistung schriftlich getroffen werden. Sie unterliegt Formvorschriften (siehe Formular-Beispiel). Sie muss expressis verbis die Feststellung enthalten, dass eine Erstattung der Vergütung durch Erstattungsstellen möglicherweise nicht in vollem Umfang gewährleistet ist. Nur Notfall- und akute Schmerzbehandlungen dürfen nicht in einer Honorarvereinbarung abgebildet werden. Es ist bei der Honorarvereinbarung einerlei, ob der Patient privat versichert ist, oder ob es sich um eine nach den Bundesmantelverträgen korrekt vereinbarte Privatleistung beim gesetzlich krankenversicherten Patienten handelt.

Häufige Leistungen, die von der krassen Unterhonorierung betroffen sind, sind beispielsweise:

- HKP bei KFO oder Funktionsanalyse (GOZ-Nr. 0040)

- Fluoridierung (GOZ-Nr.1020)

- Plastischer Aufbau vor ZE (GOZ-Nr.2180)

- Pädiatrische Krone (GOZ-Nr.2250)

- Wiedereingliederung Inlay etc. (GOZ-Nr.2310)

- Exzisionen (GOZ-Nr.3070, 3080)

- Plastischer Kieferhöhlenverschluss (GOZ-Nr.3090)
- Beseitigen störender Schleimhautbänder (GOZ-Nr.3210)

- Wundrevision (GOZ-Nr.3310)

- Parodontalstatus (GOZ-Nr.4000)

- Adhäsivbrücke (GOZ-Nr.5150)

- Teilunterfütterung (GOZ-Nr.5270)

- Entfernung eines Bandes (GOZ-Nr.6130)

Es gibt keinen Grund, die Überlegungen des Bundesverfassungsgerichtes nicht alltäglich in der eigenen Praxis bei dem gemeinhin gegebenen Leistungsstandard gängig zu machen.

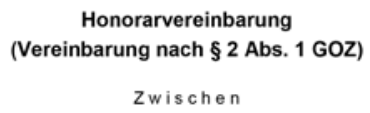

Praxis

Herr/Frau

ggf. Vorname, Name

Straße, Hausnummer

PLZ, Ort

Patient/in

und

Frau/Herr (Zahlungspflichtiger oder gesetzlicher Vertreter)

Vorname, Name

Straße, Hausnummer

PLZ, Ort

Abweichend von der Gebörenordnung für Zahnărzte (GOZ) vereinbaren die Parteien für folgende Leistungen die nachfolgend aufgefuhrten Gebahren:

\begin{tabular}{|l|l|l|l|l|l|}
\hline $\begin{array}{l}\text { Zahn/Gebiet/ } \\
\text { Region }\end{array}$ & $\begin{array}{l}\text { Gebührennummer } \\
\text { (GOZ/GOA) }\end{array}$ & $\begin{array}{l}\text { Bezeichnung der } \\
\text { Leistung }\end{array}$ & $\begin{array}{l}\text { Steigerungs- } \\
\text { satz }\end{array}$ & Anzahl & $\begin{array}{l}\text { Betrag } \\
\text { in EUR }\end{array}$ \\
\hline & & & & & \\
\hline & & & & & \\
\hline & & & & & \\
\hline Summe & & & \\
\hline
\end{tabular}

Es wurde ausdrūcklich darauf hingewiesen, dass eine Erstattung der Vergütung durch Kostentrăger bzw. Erstattungsstellen möglicherweise nicht in vollem Umfang gewâhrleistet ist. Die Parteien sind sich darüber einig, dass die Zahlung des vereinbarten Honorars hiervon unabhăngig zu erfolgen hat. Dem Zahlungspflichtigen (oder gesetzlichen Vertreter) wurde eine Ausfertigung dieser Vereinbarung ausgehändigt.

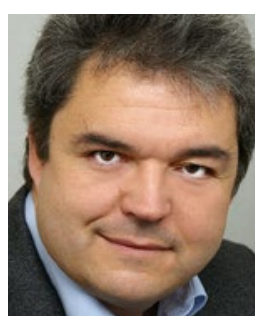

Dr. Dr. Alexander Raff

Mitglied im GOZ-Expertenrat des FVDZ 